\title{
INVESTIGACION
}

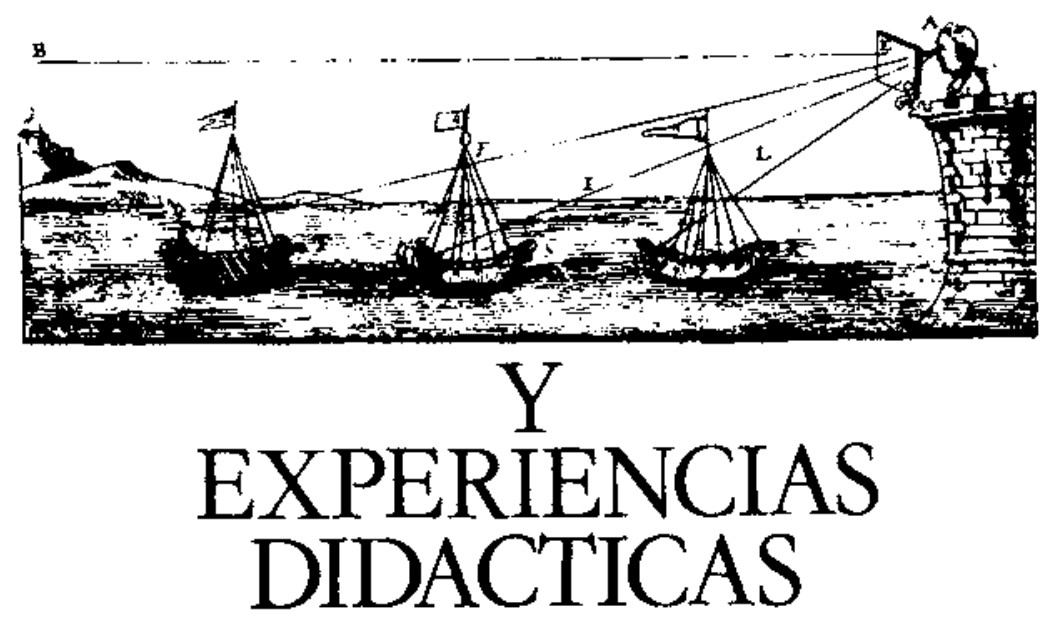

\section{APRENDIZAJE POR DESCUBRIMIENTO: PRINCIPIOS Y APLICACIONES INADECUADAS}

\author{
BARRÓN RUIZ, A. \\ Universidad de Salamanca. Facultad de Filosofia y Ciencias de la Educación. Paseo de Canalejas, 169. \\ 37008 Salamanca.
}

\section{SUMMARY}

This article deals with a critical analysis of the practical applications of discovery learning. To this end, the principles on which we have reconstructed that theory of learning are used, according to a constructivist foundation of cognitive activity as a conjectural activity, mediated by sociocultural orientations. This article is based on previous research by the author (Barrón 1989, 1991a, 1991b). After defining and establishing the theoretical principles, the inadequate practical applications of discovery learning are examined, derived, in some cases, from an empiricist concept of discovery, and in others, from a «scientific reductionism», «individualist», or «mechanistic» concepts of learning by discovery.

Este trabajo está centrado en el análisis de los principales errores que suele conllevar la práctica escolar del aprendizaje por descubrimiento, de acuerdo con los principios teóricos sobre los que hemos reconstruido dicha teoría de aprendizaje (Bartón Ruiz 1991).
I. PRINCIPIOS DE LA TEORÍA DEL APRENDIZAJE POR DESCUBRIMIENTO

Empezaremos por sintetizar una definición del aprendizaje por descubrimiento, entendido como actividad au- 
torreguladora de resolución de problemas, que requiere la comprobación de hipótesis como centro lógico del acto de descubrimiento. Pasamos seguidamente a exponer los principios.

\section{EI ser humano está dotado de potencialidad natural para descubrir conocimiento}

El sujeto del descubrimiento es una totalidad sistémica y comunicativa, dotado de capacidad para autorregular su comportamiento, gracias a lo cual puede desarrollar experiencias de aprendizaje por descubrimiento.

Al caracterizarlo como totalidad queremos recalcar que en el proceso de aprendizaje participa el sujeto integral, no sólo sus aspectos intelectivos, sino tambiér afectivos, psicomotores, morales, sociales... La capacidad de autorregulación se desarrolla cuando el sujeto aplica sus sistemas cognitivo, comprensivo y actuacional, mediante los que el sujeto interpreta la realidad, elabora sus expectativas, objetivos... y autorregula su intervención.

\section{El resultado del descubrimiento es una construcción intrapsíquica novedosa}

El resultado del descubrimiento es una «construcción» intrapsíquica novedosa para el sujeto, aunque no lo sea para la colectividad social. Se trata de un «descubrimiento asimilativo", que implica la reconstrucción de un significado novedoso para su sistema cognitivo. En tanto que construcción, todo descubrimiento emerge de una serie de conocimientos previos, que son diferenciados y coordinados con nuevos elementos, para configurar significados novedosos (Piaget 1970, Kelly 1955, Claxton 1987, Watzlawick et al. 1989, Barrón 1991b...).

\section{El aprendizaje por descubrimiento encuentra su punto de partida en la identificación de problemas}

La epistemología postpositivista ha fundamentado la actividad cognoscitiva como actividad conjetural que encuentra su punto de partida no en la observación sino en Ia aplicación de expectativas. Cuando las expectativas del sujeto resultan frustradas, o son insuficientes para conseguir un objetivo, se configuran situaciones problemáticas que pueden desencadenar el proceso de indagación y descubrimiento (Popper 1983, Kuhn 1975, Hanson 1977, Lakatos 1983, Laudan 1977, Simon 1977, Goldman 1983...). Como ya planteara Dewey (1933), es del enfrentamiento con la dificultad, con la incertidumbre, con el problema..., de donde emerge el proceso reflexivo que obliga a extender, diferenciar, reformular las teorías previas, para configurar otras nuevas.

\section{El aprendizaje por descubrimiento se desarrolla a través de un proceso de resolución significativa de problemas}

Desde el punto de vista procesual, podemos definir el aprendizaje por descubrimiento como un proceso de resolución significativa de problemas, basado en la disposición intencional del sujeto hacia la comprobación de hipótesis que incorporen una comprensión de la relación medios-fin, fundamentadora del descubrimiento (Ausubel et al. 1983, p. 485). Como tal, no procede por secuencias inductivas derivadas de la observación, sino a través de un proceso constructivo de comprobación de teorías, basado en la coordinación de acciones que el sujeto ejerce sobre la situación problemática planteada.

\section{El acto de descubrimiento encuentra su centro lógico en la comprobación de conjeturas}

Si bien el proceso de descubrimiento obedece a regularidades de conceptualización y comprobación de hipótesis, y si bien ambas se necesitan mutuamente, es el criterio de comprobación el que determina el centro lógico del concepto de descubrimiento. Así, mientras pueden existir descubrimientos basados en la comprobación de hipótesis planteadas por otros, no pueden existir descubrimientos basados en la mera concepción đe hipótesis si éstas no resultan probadas (Barrón 1991a, pp. 158-163).

6. Para que la actividad resolutiva pueda ser caracterizada de descubrimiento ha deser autorregulada y creativa

En el aprendizaje por descubrimiento, el sujeto ha de autorregular el procedimiento de resolución, sobre todo en lo relativo a la fase de comprobación. Respetar la autorregulación implica que sea el sujeto quien elija, organice, aplique y controle la secuencia de operaciones que componen las estrategias de comprobación. La resolución no podrá ser calificada de descubrimiento si únicamente implica la reproducción de conocimientos ya poseídos o la aplicación de algoritmos proporcionađos. Además de ser autorregulada, requiere la utilización del pensamiento productivo y la construcción de un hallazgo intrapersonal novedoso (Piaget 1981b, pp. 44-47).

\section{El aprendizaje por descubrimiento va asociado a la producción de errores}

La intervención instructiva tradicional ha tendido a penalizar el error o a considerarlo como algo indeseable que hay que eliminar, no prestándole mayor atención. Sin embargo, tanto la psicogénesis como la epistemología del descubrimiento demuestran la productividad cognoscitiva del error (Barrón 1989). De acuerdo con Piaget, «un error corregido puede ser más fecundo que un Éxito inmediato» (Piaget 1981a, p.114, Casavola 1983, Gil y Carrascosa 1985, Giordan 1982, Moreno 1983...). En definitiva, es la toma de conciencia del error lo que estimula la elaboración de nuevas conjeturas y la construcción de nuevos descubrimientos. Por ello, ei error ha de ser valorado positivamente e incluso, en ocasiones, promovido como recurso educativo para posibilitar el acceso a conocimientos superadores de los anteriores. 
Pasando por alto los errores de los alumnos, limitando la intervención del enseñante a la explicación de la solución correcta, estaremos fomentando aprendizajes superficiales, ya que los esquemas cognitivos del sujeto no estarán adecuadamente estructurados para integrar la nueva información.

\section{Al aprendixaje por descubrimiento le es consustancial la mediación sociocultural}

No existe experiencia cognoscitiva, por muy autónoma que sea, que no se halle mediada por orientaciones socioculturales. El aprendizaje por descubrimiento, aunque requiere el comportamiento autorregulador del sujeto, no puede ser planteado como exento de la mediación sociocultural, sino derivado de la autodirección.

A este respecto apuntamos que las experiencias colectivas y cooperativas de aprendizaje por descubrimiento, en la medida en que animan al sujeto a expresar y fundamentar su pensamiento, a descentrar su razonamiento, a coordinar su acción con la de los demás... pueden resultar altamente favorecedoras de los descubrimientos cognitivos intrapersonales (Vygotski 1979, Wertsch 1988, Perret-Clermont 1984, Forgas 1981, Nelson 1988...).

\section{El grado de descubrimiento es inversamente proporcional al grado de predeterminación del proceso resolutivo}

La necesidad del descubrimiento se presenta alli donde el conocimiento no viene directamente determinado por indicaciones externas ni por recursos internos disponibles, en el sentido de mera reproducción de algo suministrado o existente. En el momento en que existan indicaciones (externas o internas) que determinen directamente el procedimiento resolutivo, anulando la necesidad de actividad autorreguladora de investigación, se habrá anulado asimismo la posibilidad de la experiencia cognoscitiva de descubrimiento.

\section{El aprendizaje por descubrimiento puede ser} pedagógicamente promovido

Recogiendo la línea de pensamiento que defiende la posibilidad de una lógica del descubrimiento, entendemos que el descubrimiento no se explica por la intervención de hechos no controlables, poderes especiales, ni fases o estadios irracionales. En buena medida, el proceso de descubrimiento se fundamenta en «estructuras actitudinales frente a los datos en vistas a su configuración en problemas; frente al problema, para el planteamiento de conjeturas; frente a las conjeturas o hipótesis, en vistas al diseño de programas de experimentación y comprobación» (García Carrasco 1983, p.16, Dewey 1950, Hanson 1977, Hintikka 1981. Agassi 1981, Jarvie 1981, Nickels 1980, Simon 1973...).

Para M. Bunge, el proceso de invención y descubrimiento «no es ilegal», sino que sigue ciertas pautas» (Bunge 1984 , p. 48 ); pero «no es un procedimiento mecaniza- ble» porque requiere originalidad y «la mera idea de un conjunto de prescripciones para conseguir la originalidad es autocontradictoria» (Bunge 1973, p. 497). Los procesos que han conducido a los grandes descubrimientos científicos han estado basados en la alta capacidad de integración y estructuración operativa, sobre un amplio y sistemático basamento de conocimientos adquiridos en torno al tema de investigación (Mansfield y Busse 1981, Michie y Johnston 1984, Gruber 1984, Ausubel et al. $1983 \ldots)$.

Por otra parte, no hay que olvidar que el comportamiento creativo, al igual que cualquier otro aspecto de la conducta humana, si bien se asienta sobre potencialidades innatas, adquiere su configuración como fenómeno de naturaleza social y por tanto educable, debiendo considerar el importante papel que puede tener el comportamiento investigador y creativo del profesor como modelador del comportamiento de sus alumnos y la importancia que tiene la interacción grupal del aula en el favorecimiento del descubrimiento, al activar en el sujeto la búsqueda de sintesis creativas de las confrontaciones derivadas de la interacción social.

\section{USOS INADECUADOS DEL APRENDIZAJE POR DESCUBRIMIENTO}

\section{Concepción empirista del descubrimiento}

Cuando a mediados del siglo $\mathrm{XX}$ se desencadena la «moda» del aprendizaje por descubrimiento, sobre todo en el ambiente norteamericano, se inicia una importante controversia en torno a la problemática de la enseñanza y del aprendizaje escolar. Subyaciendo a tal controversia se alimenta una categorización empirista del descubrimiento, que tiende a ser identificado como un aprendizaje basado en la experiencia empírica, en oposición al aprendizaje teórico y verbalista tradicional.

Aparece así una concepción del descubrimiento que sitúa la fuente del mismo en la experiencia sensible y el dato perceptivo, y cuyo desarrollo se halla fuertemente asociado a secuencias de aprendizaje inductivo, en tas que, partiendo de la observación de la realidad y por medio de procesos de ensayo-error, pensamiento intuitivo y abstracción empírica, se llega al descubrimiento de generalizaciones cognitivas.

\section{I. Identificación del descubrimiento con secuencias de aprendizaje inductivo}

La controversia entre aprendizaje receptivo y aprendizaje por descubrimiento se encuentra asociada, desde sus comienzos, con la controversia sobre métodos deductivos (regla-ejemplo) / métodos inductivos (ejemplo-regla). Recogiendo las ideas de Bruner (1961), Glaser (1974), Cronbach (1975)..., diversos autores identifican el aprendizaje por descubrimiento con secuencias inductivas, que tienen su punto de partida en la observación de datos particulares, a partir de las cuales el sujeto llega a 
descubrir la generalización subyacente (Beltrán 1987, p. 81 , Muñoz 1985, p. 470, Tomlinson 1984, p. 170, Blázquez Entonado et al. 1983...).

En el pensamiento educativo de muchos profesores se ha constatado la existencia del falso dilema empirismo/ teoricismo, advirtiéndose que sitúan el punto de partida del proceso cognoscitivo en la observación, para continuar con el planteamiento de hipótesis, su experimentación y posterior abstracción. Aunque las aportaciones de la Psicología Cognitiva y de la Epistemología Postpositivista fundamentan que el avance del conocimiento procede hipotéticamente-deductivamente, en la actividad escolar subyacen diferentes asunciones que tienden a favorecer procedimientos inductivos.

Harris y Taylor (1983, pp. 280-281) sugieren algunos factores que explican tal disparidad. En primer Iugar, la simplicidad de las tareas escolares en las que, a los alumnos, se les pide que investiguen un fenómeno sin atender al estado de sus teorías previas y a la actividad central de comprobación de hipótesis. En segundo lugar, la larga tradición pedagógica existente en defensa de los procedimientos inductivos. Cuando Rousseau estableció la sabiduría del «desenvolvimiento natural», introdujo una filosofía inductivista en educación que alentó todo el movimiento de la educación progresista de principios del siglo XX. Desde Pestalozzi, Froebel, Montessori y hasta el propio Bruner, se ha insistido más en que los alumnos observen y experimenten que en la necesidad de que comprueben sus hipótesis.

Un análisis de los criterios metodológicos promovidos por los profesores de ciencias durante los dos primeros años de la Reforma de las Enseñanzas Medias (años 8486) en España demostró que:

-Existe un rechazo al modelo transmisivo de la enseñanza tradicional, pero no hay una formulación clara de una propuesta altemativa.

- Se insiste en la aplicación del método científico y el aprendizaje por investigación y descubrimiento; pero las alternativas metodológicas son diversas, insuficientemente delimitadas y fuertemente marcadas por secuencias inductivas que inician las tareas de aprendizaje a través de la observación y el contacto con la realidad, basándose en la creencia de que los alumnos podrán llegar a abstraer el conocimiento necesario.

- Escasean las actividades de formulación y comprobación de hipótesis (Pedrinaci 1986, pp. 223-228, Gil 1983 , p. 27 , Hodson 1988, pp. 5-21, Porlán 1988, pp. 193-203...).

Las creencias empiristas han alimentado la consideración del aprendizaje por descubrimiento como aprendizaje inductivo, propiciando la estructuración de la actividad escolar en base a:

- Experiencias empíricas (actividades de exploración, observación y manipulación) que permitan el contacto con los datos del problema, desatendiendo los conocimientos previos del alumno.
- Organización externa del proceso resolutivo, con el fin de orientarlo hasta el estado meta, desatendiendo la actividad estructurante del alumno.

La fundamentación epistemológica de la existencia de expectativas teóricas como elementos configuradores de los enunciados observacionales ha reconceptualizado la imagen de la actividad cognoscitiva como actividad conjetural. La activación del proceso cognoscitivo no se encuentra en los datos empíricos, sino en los esquemas interpretativos del sujeto: lo importante no es proporcionar experiencias empíricas, sino la relación que éstas pueden entablar con los esquemas asimilativos del atumno.

El sujeto nunca parte de cero en su interacción con el medio. Como expresan Ausubel y col., «raro es el caso en que un individuo ataca un problema sin ninguna hipótesis general para dirigir la interpretación de los datos. Resulta, pues, una burda y exagerada simplificación insistir en que cuando se piensa inductivamente, se va de los casos particulares a las generalizaciones, y que las hipótesis se formulan exclusivamente a partir de los datos» (Ausubel et al. 1983, $2^{\natural}$ ed., p. 490).

De acuerdo con el principio tercero, anteriomente mencionado, el aprendizaje por descubrimiento no encuentra su punto de partida en la observación, sino en el enfrentamiento con problemas, los cuales emergen cuando las expectativas del sujeto resultan frustradas o son insuficientes para entender una situación o conseguir un propósito.

\subsection{Usos inadecuados derivados de ignorar la impor- tancia de la "comprobación" en la configuración del descubrimiento}

En la tradición epistémica positivista, el đescubrimiento suele quedar identificado con el episodio heurístico por el que se concibe una hipótesis, estableciéndose una independencia entre el contexto de descubrimiento y el contexto de justificación. Es la misma epistemología la que subyace a muchas de las experiencias pedagógicas de aprendizaje por descubrimiento, que han sido planteadas como fomento de actividades đe libre exploración, manipulaciones espontáneas, contacto empírico con la realidad...; todo ello orientado a promover en el sujeto la abstracción de generalizaciones, sin continuar después con la justificación de las mismas.

Con tales experiencias, los alumnos adquieren una aproximación intuitiva al conocimiento pero carecen de descubrimientos claros y significativos, los cuales, al igual que los descubrimientos científicos, no constituyen una mera prolongación de la generalización inductiva, sino que han de ajustarse a las restricciones lógicas que impone la comprobación. Atendiendo al principio quinto, hay que teneren cuenta que el acto de descubrimiento tiene su centro lógico en la verificación de conjeturas. Sin embargo, en determinadas prácticas escolares, se ha operado sobre la suposición incorrecta de que la concepción de una hipótesis acertada podría ser considerada como un descubrimiento hecho por el alumno, cuando en realidad el estudiante que formula una hipótesis no 
descubre nada hasta que no obtiene alguna evidencia que pruebe la corrección de su suposición. En este sentido, las intervenciones docentes que refuezan una hipótesis acertada con un entusiasta «eso es» puede que tengan sus virtudes pedagógicas, pero lo que también hacen es truncar el proceso de descubrimiento, al eliminar el momento clave de la comprobación.

La concepción empirista ha favorecido la identificación del aprendizaje por descubrimiento con una experiencia intuitiva, asentada en el contacto directo del sujeto con el material de conocimiento, de manera que pueda llegar a una cognición inmediata a través de los sentidos. Desde esta perspectiva, se tratará de sustituir la transmisión verbal por el contacto con la realidad, bien se trate de un contacto directo con el objeto o indirecto, promovido a través de representaciones icónicas (uso de medios au diovisuales, modelos, imágenes...). Figuras clásicas como Locke, Rabelais, Montaigne, Comenio, Rousseau, Pestalozzi, Decroly ... podrian ser representantes de tal creencia. Sobre tal fundamento, los métodos intuitivos sustituyen el predominio de la palabra por el predominio de la imagen.

Piaget considera como fuente inspiradora de tales propuestas empiristas a la Psicología de la Gestalt, que tanta importancia ha concedido a las estructuras perceptivas (gestalts) y a los fenómenos intuitivos de insight. Bajo los métodos intuitivos subyace la suposición epistémica errónea de concebir el conocimiento como copia figurativa de la realidad, a través de registros perceptivos. Sin embargo, el conocimiento no constituye una copia de la realidad, sino una asimilación de lo real a estructuras de transformaciones: «Conocer es asimilar lo real a estructuras de transformaciones, siendo estas estructuras elaboradas por la inteligencia en tanto que prolongación directa de la acción» (Piaget 1981b, p. 38).

El aprendizaje por descubrimiento involucra evidentemente la aplicación del pensamiento intuitivo que per. mite al sujeto una captación rápida y global del espacio del problema y del camino a seguir; pero, en la medida en que todo descubrimiento ha de estar apoyado en pruebas o razones que lo validen como tal, es necesario la utilización de métodos analíticos más rigurosos.

En las experiencias de aprendizaje por descubrimiento, el enfrentamiento intuitivo tiene especial relevancia en la fase inicial del proceso, orientándose hacia la comprensión global del problema y formulación đe propuestas iniciales de resolución.

Promover la expresión de las ideas intuitivas acerca de una situación problemática es un paso pedagógicamente necesario para iniciar la experiencia resolutiva del descubrimiento, ya que proporciona las concepciones cognitivas discentes, de las que el docente deberá partir, y sobre las que deberá intervenir en la orientación del proceso; pero la secuenciación de la experiencia descubridora obliga a transcender tal enfrentamiento intuitivo y estimular la construcción de descubrimientos cognitivos mejor fundados. Como expresara Bruner, la «intuición, para ser fructífera, debe tener este sentido de incompleto, la sensación de que queda algo más por hacer», porque «a menos que esté sincronizada con métodos más rigurosos y unificados, su deficiencia es una mezcla de imprecisión e incorregibilidad» (Bruner 1987, p. 99).

\subsection{Interpretación del aprendizaje por descubrimiento como aprendizaje por ensayo y error}

Sobre la base de una epistemología empirista es frecuente asimismo haber identificado al aprendizaje por descubrimiento con la naturaleza de un aprendizaje por «ensayo y error» (Glaser 1974, Muñoz 1985, Beltrán 1987...).

EI aprendizaje por ensayo y error configura uno, entre otros, de los procedimientos que puede adoptar el sujeto cuando se enfrenta a un problema. Bruner (1961, pp. 21 . 32) caracterizó una dimensión cognitiva, subyacente al comportamiento indagador, cuyos polos representarían, por una lado, la ciega resolución de ensayo y error (empirismo episódico) y, por otro, la resolución comprensiva de descubrimiento (constructivismo acumulativo). La disposición resolutiva del empirismo episódico se caracteriza por: a) disposición a creer que no existe ninguna relación significativa por descubrir; $b$ ) búsque. da azarosa de información, sin orientación hacia la formulación de hipótesis; c) recogida arbitraria e inconexa de información; $d$ ) falta de persistencia organizada; e) resultados infructuosos y aprendizajes no significativos.

En contraposición, el polo del «constructivismo acumulativo» (cumulative constructionism) vendrá caracterizado por: a) disposición a creer que existe alguna regularidad significativa medios-fin, por descubrir; $b$ ) búsqueda de información orientada hacia la formulación y comprobación de hipótesis; c) recogida de información de forma organizada y relacionada; $d$ ) persistencia organizada; $e$ ) descubrimientos satisfactorios y aprendizajes significativos.

Ante tales disposiciones resolutivas, Bruner resalta que Ia actividad cognitiva que subyace al aprendizaje por descubrimien to está mayormente inclinada hacia el polo del «constructivismo acumulativo».

Ausubel y col. (1983) se refieren al aprendizaje por ensayo y error como una resolución «ciega», basada en la disposición a variar las respuestas por aproximación y correcciones aleatorias hasta que aparezca una variante acertada, sin existir comprensión ni integración significativa de la relación causal medios-fin. En contraposición con tal modalidad, distinguen el aprendizaje por "discernimiento», caracterizado por una resolución "comprensiva" basada en la disposición hacia la formu* lación y comprobación de hipótesis que incorporen una relación del principio medios-fin susceptible de fundamentar la resolución del problema. Mientras el «discernimiento" produce aprendizajes significativos, la resolución por ensayo y error produce internalizaciones arbitrarias.

Como hemos establecido en el principio cuarto, el apren* dizaje por descubrimiento se desarrolla a través de un proceso de resolución significativa de problemas. El sujeto que descubre por ensayo y error encuentra la 
solución «por casualidad»: no sabe a qué ha sido debido, ni sabría cómo repetirlo. Y como ya hemos fundamentado, no se puede afirmar haber descubierto algo sin tener razones para probarlo, aunque sólo sea funcionalmente, como lo prueban los niños que repiten una y otra vez sus pequeños descubrimientos.

Con la distinción realizada no se quiere indicar que en el comportamiento subyacente al descubrimiento no existan procedimientos de ensayo y error. En muchos problemas no queda más remedio que proceder de un modo más cercano al ensayo y error que al discernimiento, sobre todo cuando no resulta discernible ningún patrón de relaciones significativas, bien porque se trata de condiciones arbitrarias o porque el sujeto carece de conocimientos en la temática. Igualmente sucede con la investigación científica revolucionaria, en la que los problemas configuran dominios escasamente explorados, para cuya resolución se utilizan métodos débiles de ensayo y error (Simon et al. 1981, Larkin et al. 1980).

En este sentido, para no exponer a los alumnos a situaciones resolutivas en las que tengan que proceder por ensayo y error, hay que tener en cuenta la importancia pedagógica que tiene la fase previa de «preparación», en la que el docente ha de establecer los antecedentes necesarios para que el alumno pueda desarrollar una actividad significativa de descubrimiento. Identificar las experiencias de descubrimiento con aprendizajes por ensayo y error libera al profesor de la responsabilidad de tener que preparar y orientar adecuadamente el proceso resolutivo, y condena al aiumno a procesos de aprendizaje lentos, ciegos e infructuosos.

\section{Reduccionismo «cientista» en la concepción del descubrimiento}

La identificación del aprendizaje por descubrimiento como la aplicación al aula del método científico ha conllevado implicaciones pedagógicas inadecuadas, por la falsedad de la premisa de partida, ya que las experiencias escolares de descubrimiento no constituyen experiencias propiamente científicas sino cognoscitivas. Pretender que los alumnos jueguen a ser pequeños científicos puede conducir a las siguientes situaciones ambiguas.

- Por parte del profesor, tener que fingir que él no sabe la respuesta, o la situación paradójica de enseñar algo que todavía él no ha aprendido, e implicarse como un "socio» de la investigación, desconocedor del resultado, cuando es precisamente tal conocimiento el que le proporciona el control de la situación y la posibilidad de orientar el comportamiento de los alumnos.

- Los alumnos, por su parte, tlegan a ser conscientes de que el profesor conoce la respuesta final, y está intentando hacerles trabajar para que lleguen a descubrirla, lo cual puede hacer que la situación adquiera las connotaciones de un juego de acertijos, en el que los alumnos adquieren habilidades para sacar la información al profesor a base de múltiples trucos, con la única intención de fingir que descubren y con ello satisfacer las expectativas del profesor.

Tales peligros pueden ser reducidos si se clarifica que el descubrimiento escolar es un descubrimiento asimilativo, que se desarrolla en un contex to diferente al de la comunidad científica, utiliza instrumentos y proce " sos cognitivos diferentes y pretende objetivos distintos. No configura, por tanto, una experiencia científica, aunque tenga a ésta como modelo de referencia, sino una experiencia cognoscitiva, y como tal ha de ser presentada a los alumnos; de lo contrario, estaremos introduciendo premisas falsas que pueden predisponer hacia el mantenimiento de simulacros de descubrimiento.

Como hemos establecido en los dos primeros principios, el ser humano dispone de una potencialidad natural para descubrir conocimiento, pero el sentido del aprendizaje por descubrimiento en el aula es el de un descubrimiento asimilativo. Se trata de una experiencia de aprendizaje, no una experiencia científica, y como tal implica la reconstrucción de un significado novedoso para el sistema cognitivo del sujeto.

Por otra parte, plantear las experiencias de aprendizaje por descubrimiento como aplicaciones del método científico ha conducido a planteamientos erróneos tales como: a) creencia de que se trata de que los alumnos reproduzcan el mismo proceso seguido por los científicos en la historiografía del descubrimiento; $b$ ) programación de la secuencia de descubrimiento conforme a los pasos seguidos en el descubrimiento científico; c) creencia en la imposibilidad de aplicar el aprendizaje por descubrimiento a la enseñanza primaria, dada la carencia del pensamiento hipotético-formal.

Estos planteamientos pueden ser criticados por presentar una interpretación restringiday parcial (reduccionismo cientista) del comportamiento investigador. Subyace a eilo la identificación del concepto de investigación con el de investigación científica, desestimando la importancia de otros múltiples tipos de investigación ordinaria, con caracteres diferentes a los de la ciencia formal.

El comportamiento investigador refiere un conjunto amplio y diverso de estrategias de resolución de problemas, que tiene su origen en la conducta adaptativa básica de exploración y adquiere las connotaciones características del funcionamiento intelectivo de cada período evolutivo; pueden distinguirse diversos procedimientos de investigación: desde la investigación sensomotora a la intuitiva, para pasar a la investigación por medio de operaciones concretas, y llegar al control formal-abstracto del proceso de resolución. La ciencia podrá ser introducida en el aula a lo largo de lo que Bruner denomina «un currículo en espiral», que posibilite un acercamiento progresivo en función de las posibilidades cognoscitivas de cada período evolutivo. Introducir la ciencia experimental en la enseñanza primaria exigirá una configuración intuitiva o precientífica de la misma (Arrieta 1985, Kami y Devries 1983, Abruscato 1988, Tonucci y Rusca 1986, DES 1985, Woolnough $1990 \ldots)$. 


\section{Concepción individualista del descubrimiento}

La categorización del «aprendizaje por descubrimiento autónomo» plantea que ha de ser el sujeto quien llegue, por sí solo, al descubrimiento requerido. Tiene sus antecedentes en los planteamientos naturalistas de Rousseau y en su propuesta de una educación negativa que ređujera la intervención del adulto con el fin de no perturbar el proceso de maduración espontánea.

Consideramos que, en ocasiones, la espontaneidad y autonomía del aprendizaje por descubrimiento ha constituido un problema mal planteado, pues se ha apoyado sobre una concepción individualista del aprendizaje, alimentada por la tradición psicológica piagetiana y centrada en la relación cognoscitiva sujeto-objeto.

En realidad no existe experiencia alguna de aprendizaje que no se encuentre ligada y apoyada en la mediación sociocultural; mucho más cuando el marco donde se desenvuelve es la institución escolar. Como ha fundamentado la Escuela de Vygotski, los aspectos «sociocomunicacionales» son factores conformadores tanto de la naturaleza del sujeto, como de los objetos, así como del proceso de apropiación del conocimiento. La tesis del origen sociohistórico de los procesos mentales defiende que las funciones psíquicas son el resultado de la interiorización de acciones interpersonales (Vygotski 1979, p. 92, Luria 1980, Leontiev $1983 \ldots$.. .

La espontaneidad no hay que buscarla en la ausencia de mediación social, sino en la capacidad del sujeto para desarrollar un comportamiento autodirigido: «Se ha pensado a veces que la aceptación del carácter espontáneo del desarrollo mental es incompatible con el principio determinista, según el cual el desarrollo está condicionado socialmente (...) Esta concepción se apoya (...) en interpretaciones idealistas de la espontaneidad del desarrolio (...) La consideración materialista dialéctica (...) ve en cambio el carácter "espontáneo" del desarrollo como consecuencia de su automovimienton (Kostiuk, en Luria et al. 1973, pp. 56-57).

Como planteábamos en el principio octavo, el aprendizaje por descubrimiento no puede ser concebido como exento de mediación sociocultural, sino derivado de la autođirección del comportamiento. Se trata de una experiencia de naturaleza social, que puede ser pedagógicamente promovida (principio décimo), ya que obedece a las regularidades de un proceso autorregulado de comprobación de conjeturas, condicionado por una serie de variables intrapersonales y situacionales (Barrón 1991a, pp. $579-210$ ).

Desencadenar en el alumno sus procesos cognoscitivos espontáneos no se consigue con el absentismo y la no intervención, sino sabiendo hacer estimulantes y productivos los problemas y modelos de comportamiento que se presenten al sujeto. La autoeducación se hace factible en virtud de la heteroeducación. En este sentido, habrá que resemantizar el aprendizaje por descubrimiento escolar en un contex to interactivo de comunicación, tanto profesor-alumno/s como alumno-alumno/s.

\section{Concepciones mecanicistas del aprendizaje por descubrimiento}

La aplicación escolar del aprendizaje por descubrimiento ha significado, en ocasiones, transmitir el procedi. miento de resolución de un problema y permitir que sean los alumnos, de modo individual o por grupos, quienes reproduzcan dicho saber en la resolución de problemas semejantes. Se trata de las clásicas experiencias en las que el profesor explica la resolución de problemas-tipo, y pernite que los alumnos apliquen el conocimiento transmitido en situaciones análogas. El alumno se limita a reprođucir «recetas» de resolución, generando resultados correctos que, sin embargo, no constituyen descubrimientos significativos, como muestra su escasa transferencia más allá de las situaciones escolares con las que han quedado asociados.

Evaluando críticamente tales planteamientos podemos apreciar que la innovación introducida con respecto a la metodología expositiva es meramente formal, ya que no se replantea la actividad cognoscitiva desarrollada, que sigue siendo externamente regulada (no autorregulada). En realidad, se trata de pseudoexperiencias de aprendizaje por descubrimiento: experiencias reproductoras que han intentado adecuarse a la moda del «descubrimiento», sin cambiar sus convicciones y hábitos tradicionales.

Otras estructuraciones mecanicistas del proceso de descubrimiento pueden consistir en:

a) Modalidades del método socrático, en base a una secuencia de preguntas estructurada por el profesor. En este caso se estima que tales preguntas estimulan la investigación personal y conducen el razonamiento co. lectivo de la clase al descubrimiento propuesto.

b) Programación del descubrimiento a través de algoritmos o programas de ordenador, proporcionando al alumno la secuencia de acciones y operaciones que ha de desarrollar, junto con correcciones y feedback necesario para que, en su aplicación y corrección, llegue al descubrimiento pretendido.

En estos casos se parte de la consideración básica de que la condición imprescindible para el aprendizaje por descubrimiento es no proporcionar el conocimiento ela* borado al sujeto, sino potenciar que lo vaya descubrien. do a través de la aplicación de una secuencia determina* da de operaciones. La deformación aparece cuando se introduce la estructuración mecanicista de la secuencia (fuertemente influida por presupuestos conductistas) en base a: 1) el docente divide el contenido a descubrir en operaciones parciales que el alumno ha de ir realizando para aproximarse al resultado final, 2) el alumno va respondiendo y ejecutando la secuencia de operaciones externamente prefijada, hasta culminar en el resultado final.

De esta manera, el alumno llega a descubrir un conocimiento que no le ha sido proporcionado en su forma elaborada; y gracias a la dirección externa, el proceso resulta rápido y eficaz, superando las clásicas limitaciones atribuidas a la metodología del descubrimiento, en 
tanto que proceso lento y con alta probabilidad de errores. Sin embargo, como anteriormente hemos planteado, el aprendizaje por descubrimiento va asociado a la producción de errores (principio séptimo), pero el error ha de ser valorado positivamente, ya que es la conciencia del error lo que estimula la reconstrucción del conocimiento.

Lo que suele pasar en los ejemplos anteriores es que, si bien los alumnos responden satisfactoriamente a cada uno de Ios pasos prefijados y llegan a enunciar el descubrimiento final, no asimilan la estructura global del razonamiento por lo que son incapaces de reconstruirlo posteriormente.

El maestro confía en que los alumnos, al responder satisfactoriamente, Ilegan a captar la organización y significación total del conocimiento en lugar de la mera asociación de elementos parciales. Parece que en esto subyace la suposición inconsciente de que los alumnos van a integrar la misma organización resolutiva con la que el profesor ha estructurado el proceso. Pero lo que

\section{REFERENCIAS BIBLIOGRÁFICAS}

ABRUSCATO,J., 1988.Teaching Children Science. (Englewood Cliffs: New Jersey).

AGASSI, J., 1981. The logic of scientific inquiry, en Agassi, I., Science and Society. (Dordrecht: ReideI), pp. 223-238.

ARRIETA GALLESTEGUI, J.J., 1985. La resolución de probltmas en el Ciclo Inicial de la EGB, Enseñanza de las Ciencias, núm extra, 92.

AUSUBEL, D.P. et al. 1983. Psicología Educativa. (Trillas: México. $2^{\mathrm{a}}$ ed.).

BARRON, A., 1989. Similitudes entre la psicogénesis del conocimiento en el sujeto y la historiografia del conocimiento científico: implicaciones pedagógicas, Revista Española de Pedagogía, 183, pp. 315-336.

BARRÓN, A., 1991 a Aprendizaje por Descubrimiento: Análisis crítico y reconstrucción teórica. (Ed. Universidadde Salamanca y Amarú: Salamanca).

BARRON,A., 1991 b. Constructivismoy desarollo de aprendizajes significativos, Revista de Educacion, 294, pp. 301-321.

BELTRÁN, J., et al. 1987. Psicología de la Educación. (Eudema: Madrid).

BLÁZQUEZ ENTONADO et al. 1983. El aprendizaje por descubrimiento, en Didáctica General. (Anaya: Madrid), pp. 227-234.

BRUNER, J.S., 1961. The act of discovery, Harvard Educational Revicw, 31(11), pp. 21-32.

BRUNER, J.S., 1987. La importancia de la educación. (Paidós: Barcelona). puede pasar es que, al no permitir al alumno la regulación del procedimiento resolutivo, el resultado final no sea un descubrimiento significativo, sino la aplicación de un pensamiento reproductivo, externamente regulado.

De acuerdo con el principio sexto, para que la actividad resolutiva pueda ser caracterizada de descubrimiento ha de ser autorregulada y creativa; y el grado de descubrimiento es inversamente proporcional al grado de predeterminación del proceso resolutivo (principio noveno). $\mathrm{La}$ algoritmización de las operaciones a aplicar es contradictoria con la necesidad de autorregular el proceso resolutivo, transformando el pensamiento "productivo» en un pensamiento «reproductor", que contradice el componente heurístico y creativo de la experiencia de aprendizaje por descubrimiento. Ello no implica negar la utilidad de la enseñanza programada para la promoción del descubrimiento: la clave radica en cómo se use. Usada de modo que favorezca el ejercicio del pensamiento productivo y autorregulador, en lugar de adormecer éste en la reproducción de datos e instrucciones, puede llegar a ser un valioso recurso didáctico.

BUNGE, M., 1973.La investigación científica. (Ariel: Barcelona), $3^{3} \mathrm{ed}$.

BUNGE, M., 1984. La ciencia, su método y su filosofía. (Siglo $\mathrm{XX}$ : Buenos Aires).

CASAVOLA, H., 1983. El rol constructivo de los errores en la adquisición de los conocimientos, Cuadernos de Pedagogía, 108, pp. 49-54.

CLAXTON, G, 1987. Vivir y aprender. (Alianza: Madrid).

CRONBACH, L.J., 1975. Mejoramiento de la comprensión y del descubrimiento. (Pax-Méjico: Méjico).

DES, 1985. Science 5-6: A statement of policy.(HMSO: Londres).

DEWEY, J., 1950. Lógica: teoría de la investigación. (FCE: Méjico).

DEWEY, J., 1989. Cómo pensamos. Nueva exposición de la relación entre pensamiento y proceso educativo. (Paidos: Barcelona. Edición original en 1933).

FORGAS, J. P. (ed.), 1981 . Social Cognition. (Academic Press: Londres).

GARCÍA CARRASCO, J., 1983. Ideología y Pedagogía, Enharonar, 5-6, pp. 27-50. (Universitat Autònoma de Barcelona).

GIL, D, I983. Tres paradigmas básicos en la enseñanza de las Ciencias, Enseñanza de las Ciencias, Vol. 1(1), pp. 26-33.

GIL, D. y CARRASCOSA, J., 1985. Science learning as a conceptual and methodological change, European Journal of Science Education, 7(3), pp. 231-236. 
GIORDAN, A., 1982. La enseñanza de las ciencias. (Pablo del Río: Madrid).

GLASER, R., 1974. Variables en el aprendizaje por descubrimiento, en Shulman, L.S., Keislar, E.R, Aprendizaje por descubrimiento. Evaluación crítica. (Trillas: Méjico).

GOLDMAN, A.I., 1983. Epistemology and the theory of problem solving, Synthese, 55 , pp. 21-48.

GRUBER, H.E., 1984. Darwin sobre el hombre. Un estudio psicológico de la creatividad científica. (Alianza: Madrid).

HANSON, N.R., 1977.Patrones de descubrimiento. Observación y Explicación. (Alianza: Madrid).

HARRIS, D. y TAYLOR, M., 1983. Discovery learning in school science: the myth and the reality, Journal Curriculum Studies, 15(3), pp. 277-289.

HINTIKKA, J., I981. Scientific method as a problem solving and question-answering technique. (Reidel: Dordrecht).

HODSON, D., 1988. Filosofía de la ciencia y educación científica, en Porlán, R. et al., Constructivismo y enseñanza de las ciencias. (Diada: Sevilla. Edición original en 1986), pp. 5-21.

JARVIE, I.C., 1981. The rationality of creativity, en Dutton, D. y Krausz, M., The concept of creativity in science and art. (Martinus Nijhoff: Boston), pp. 109-128.

KAMII, C. y DEVRIES, R., 1983. El conocimiento físico en la educación preescolar. Implicaciones de la teoría de Piaget. (Siglo XXI: Buenos Aires).

KELLY, G.A., 1955. The psychology of personal constructs. (Norton Company: New York).

KUHN, T.S., 1975. La estructura de las revoluciones cientificas. (FCE: Buenos Aires).

LAKATOS, I., 1983. La metodología de los programas de investigación científica. (Alianza: Madrid. Edición original en 1978).

LARKIN, J.H. et al., 1980.Instructional implications of research on problem solving. (Jossey Bass: San Francisco).

LAUDAN, L., 1977. Progress and its problems. Towards a theory of scientific growth. (Routledge \& Kegan Paul: Londres).

LEONTIEV, A., 1983. El desarrollo del psiquismo.(Akal: Madrid).

LURIA, A.R., 1980. Los procesos cognitivos. Análisis sociohistórico. (Fontanella: Barcelona).

LURIA, R.LEONTIEV, A. y VYGOTSKI, L.S., 1973. P sicología y Pedagogia. (Akal: Madrid).

MANSFIELD, R.S. y BUSSE, T.V., 1981. The psychology of creativity and discovery: scientifics and their work. (NelsonHall: Chicago).

MICHIE, D. y JOHNSTON, R., 1984. The creative computer. (Penguin: Middlesex).
MORENO, M., 1983.La Pedagogía Operatoria.(Laia: Barcelona).

MUÑOZ ARRABAL,T,, 1985. Aprendizaje por descubrimiento, en Mayor, J. (dir.), Psicología de la Educación. (Anaya: Madrid), pp. 466-484.

NELSON, K., 1988. El descubrimientodel sentido. La adquisición del significado compartido. (Alianza: Madrid).

NICKLES, Th. (ed.) 1980. Scientific discovery, logic and racionality. (Dordrecht: Reidel).

PEDRINACI, E., 1986. Criterios metodologicos en la Reforma de las Enseñanzas Medias: situación actual y perspectiva. Actas de las IV Jornadas de estudio sobre la investigación en la escuela. Sevilla, pp. 223-228.

PERRET-CLERMONT, A.N., 1984. La construcción de la inteligencia en la interacción social. (Aprendizaje Visor: Madrid).

PIAGET, J., 1970. La construcción de lo real en el niño. (Proteo: Buenos Aires. $3^{\mathrm{a}}$ ed.).

PIAGET, J., 1981a. Lo posible, lo imposible y lo necesario, en Monografia de Infancia y Aprendizaje: Piaget, núm. 2, 1981, pp. 108-121.

PIAGET, J., 1981b. La teoría de Piaget, Infancia y Aprendizaje. núm. 2, pp. 13-54.

POPPER, K.R., 1983. Conjeturas y refutaciones. El desarrollo delconocimiento científico. (Paidós: Barcelona. Ed.orig.1963).

PORLÁN, R., 1988. El pensamiento científico y pedagógico de maestros en formación, en Porlán et al., Constructivismo y enseñanza de las ciencias. (Díada: Sevilla), pp. 193-203.

SIMON, H.A., 1973. Does scientific discovery have a logic?, Philosophy of Science, Vol. 40, pp. 471-480.

SIMON, H.A., 1977. Scientific discovery and the psychology of problem solving, en Simon, H.A., Models of Discovery. (Reidel: Dordrecht), pp. 286-303.

SIMON, H. et al., 1981. Scientific discovery as probiem solving, Synthese, 47, pp. 1-27.

TOMLINSON, P., 1984. Psicología Educativa. (Pirámide: Madrid).

TONUCCI, F. y RUSCA, G., 1986.ll bambino ilmondo vivente, il bambino e la scienza. (La Nuova Italia: Firenze).

VYGOTSKI, L.S., 1979. El desarrollo de los procesos psicológicos superiores. (Grijalbo: Barcelona).

WATZLAWICK, M.W. et al. 1989. La realidad inventada. (Gedisa: Barcelona).

WERTSCH, J.V., 1988. Vygotski y la formación social de la mente. (Paidós: Barcelona).

WOOLNOUGH, B. (ed.) 1990. Practical Science. (Open University Press: Londres). 